\title{
Independence of effects of septal ablations on water intake and response inhibition
}

ROBERT J, CAREY

SYRACUSE VA HOSPITAL AND STATE UNIVERSITY OF NEW YORK UPSTATE MEDICAL CENTER

Bilateral lesions in the septal area of rats were found to independently increase water intake and operant responding for water on a DRL reinforcement schedule. These findings indicate that separate mechanisms for regulating appetitive consumatory behavior and response inhibition are present in the septal area.

Sharply conflicting data exist as to whether experimental lesions in the septal forebrain area of rats affect motivation or response inhibition. On the one hand, Harvey, Lints, Jacobson, \& Hunt (1965) and Harvey \& Hunt (1965) have reported that rats subjected to septal ablations show large and sustained increases in daily water consumption and in operant responding for water reinforcement. In addition, their studies indicated that septal lesioned rats perform in operant conditioning tasks in a way which maximizes reinforcements even if response inhibition is required to obtain the reinforcement. This was shown by the finding that septal lesioned rats obtained more reinforcements than controls when water reinforcement was programmed according to a differential reinforcement of low rates (DRL) schedule.

In contrast, however, Kaada, Rasmussen, \& Kviem (1962) found that rats subjected to septal lesions were severely impaired in a passive shock-avoidance task, but did not consume more water than controls. Furthermore, Ellen \& Powell (1964), Burkett \& Bunnell (1966), and Carey (1967) have reported that septal lesioned rats are markedly deficient in obtaining food and water reinforcement on a DRL schedule.

In order to examine further the basis for these discrepant findings, the present study correlates the effects of a large number of septal ablations on water consumption with the effects of this lesion on operant responding for water under a DRL 22-sec. schedule of reinforcement.

Thirty-seven adult male Sprague-Dawley rats weighing 400-500 $\mathrm{gm}$ and individually housed were used. Twenty-three of the rats were subjected to bilateral radio frequency lesions in the septal area. The remaining 14 animals served as controls and were subjected to sham operations in which the dura was exposed at the appropriate points, but the electrode was not introduced into the brain.

Daily water consumption was measured from the third through eighth postoperative days by simply welghing each animal's water bottle at a fixed time each day. After the final water measurements, the animals were placed on a 23-1/2-hr. water-deprivation regimen for several days and then trained to press a lever for $.1 \mathrm{cc}$ of water in an enclosed operantconditioning chamber. On the day lever pressing was shaped and on the succeeding day each animal was allowed 50 reinforcements on a continuous reinforcement schedule (CRF). This insured that the lever-pressing response was well established. On each of the following two days, each animal was placed in the test chamber for a 1-hr. period with reinforcement available on a DRL 22-sec. schedule. The number of responses emitted and the number of reinforcements obtained were recorded.

Figure 1 presents the water consumption of each animal plotted against the number of responses emitted per reinforcement in the two DRL sessions. The water consumption of each animal represents the mean intake over the last three days water consumption was measured. Inspection of Fig. 1 shows clearly that increased drinking and increased operant responding can be independently obtained from ablations in the septal area. Examination of the brains indicated that rats with high water intake and low operant responding had lesions placed deeply in the septal area which damaged the diagonal band of broca and extended posteriorly into the rostral portions of the medial preoptic area. Figure 2 shows a frontal section of the septal lesion of a rat designated by the letter " $A$ " in Fig. 1 which illustrates the deep location of the lesion in these animals. For the rats which were in the normal range for water intake, but emitted a large number of responses under the DRL schedule,

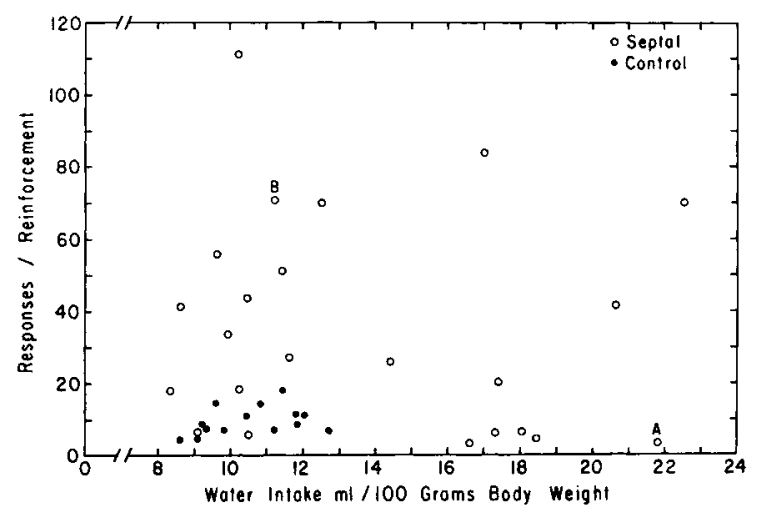

Fig. 1. Response output per reinforcement plotted against the water intake for each septal lesioned and control animal. 


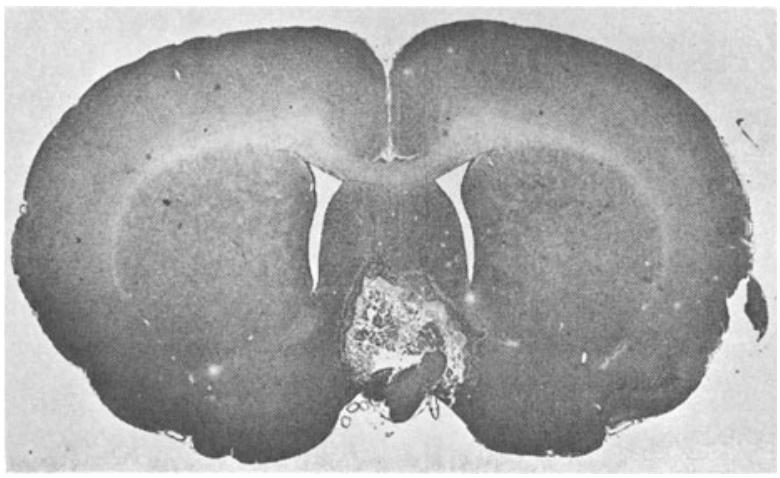

Fig. 2. Frontal section of septal lesion of the rat designated by letter " $A$ " in Fig. 1.

the lesions were more anteriorly and dorsally situated, usually at a level above the horizontal plane of the anterior commissure. Figure 3 presents a frontal section of the septal lesion of the rat designated " $B$ " in Fig. 1 which illustrates the location of this lesion. For the animals which showed both increased water intake and increased responding, the lesions generally encompassed both of these areas while the animals well within the normal range for drinking and responding sustained elther unilateral or slight bilateral lesions.

While further work is needed to delineate more

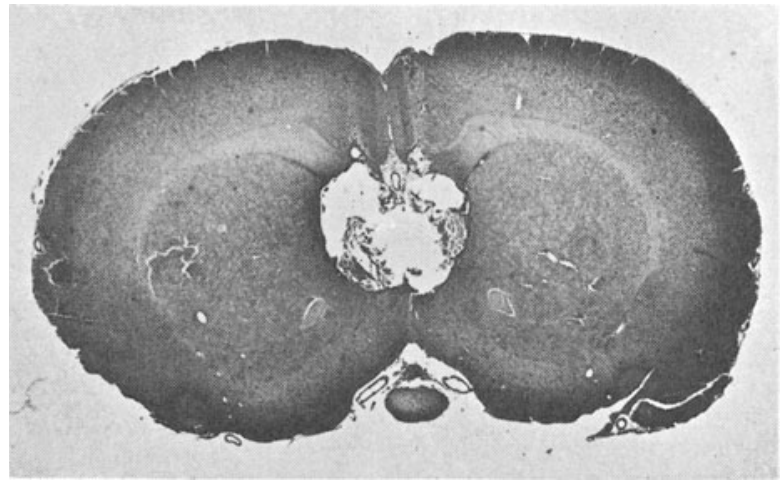

Fig. 3. Frontal section of septal lesion of the rat designated by letter " $B$ " in Fig. 1. precisely the structural damage responsible for increased water intake and repetitive responding following lesions in the septal region, the separation of these effects appears well established. These results indicate that two distinct systems exist in the septal region, one concerned with water and possibly food regulation, and the other mediating response inhibition. Thus, the previously noted discrepant findings may be attributable to the lesion having differentially affected these two systems. Furthermore, since it has been previously shown (Carey, 1967) that increased water deprivation markedly inçreases response output on a DRL 22-sec. schedule, the suggested below normal levels of operant responding evidenced by some rats (e.g., rat " $A$ " in Fig. 1) showing excessive drinking indicates that this excessive drinking does not reflect a stronger thirst motivation. While the mechanism underlying the excessive water consumption awaits clarification, the suggested dissociation between instrumental performance and consummatory behavior, in line with studies of hypothalamic hyperphagia (Miller, Bailey, \& Stevenson, 1950), further cautions against the use of modifications in consummatory behavior to infer corresponding changes in motivation.

\section{References}

Burkett, E. E., \& Bunnell, B. N. Septal lesions and the retention of DRL performance in the rat. J. comp. physiol. Psychol., $1966,62,468-472$.

Carey, R. J. Contrasting effects of increased thirst and septal ablations on DRL responding in rats. Physiol. \& Behav., 1967, in press.

Ellen, P., \& Powell, E. W. Septal inhibition and timing behavior in the rat. Exp. Neurol., 1964, 10, 120-132.

Harvey, J. A., \& Hunt, H. F. Effect of septal lesions on thirst in the rat as indicated by water consumption and operant responding for water reward. J. comp. physiol. Psychol., 1965, 59, 49-56.

Harvey, J. A. Lints, C. E., Jacobson, L. E., \& Hunt, H. F. Effects of lesions in the septal area on conditioned fear and discriminated instrumental punishment in the albino rat. $J$. comp. physiol. Psychol., 1965, 59, 37-58.

Kaada, B. R., Rasmussen, E. W., \& Kveim, O. Impaired acquisition of passive avoidance behavior by subcallosal, septal hypothalamic and insular lesions in rats. J. comp. physiol. Psychol., $1962,55,661-670$.

Miller, N. E., Bailey, C. J., \& Stevenson, J. A. F. Decreased "hunger" but increased food intake resulting from hypothalamic lesions. Science, 1950, 112, 256-259. 